\title{
Designing An Educational Game Facilitating Children's Understanding of the Development of Social Relationships using IVAs with Social Group Dynamics
}

\author{
Wan Ching Ho and Kerstin Dautenhahn \\ Adaptive Systems Research Group, School of Computer Science \\ University of Hertfordshire, Hatfield, Hertfordshire, AL10 9AB, UK \\ $\{$ w.c.ho, k.dautenhahn\}@herts.ac.uk
}

Introduction. As part of life, social relationships are fundamentally important to humans. Children are born into a family social structure and gradually they become part of a wider social structure including peers, in which each individual's relationships are affected by other relationships. To most people expressing appropriate non-verbal social behaviours when interacting with others in a virtual environment (VE) is natural and effortless as they do so daily in the physical world. There is a minority group, such as people diagnosed with autistic spectrum disorder, who encounter difficulties when carrying out this task because of impairments of specific skills regarding social communication and communication [1]. In thist paper, we study the design of a system using advanced VE and IVA technologies with the goal to help children with autism to explore and better understand social dynamics occurring in social networks involving groups of people.

Background. Synthetic agents have been widely employed to model so-called 'unconscious reactions' where agents can signal social availability and spatial awareness to others (e.g. in [2]). To achieve socially desired behaviour by agents in VE, researchers adopted models that concern the spatial distances, orientation and arrangement of agents in social situations. Specifically, the F-formation system defined by Kendon [3] and concepts on proxemics by Hall [4] are particularly suitable for virtual learning environment (VLEs).

Modelling Social Relationships in IVAs. From our previous work [5], we enhanced a VLE populated by autonomous social agents aiming at facilitating the developments of social relationships among children. A play scenario, which is similar to the context of a group of children meeting in a school playground and a canteen, involves a small number of agents embodied in a VE (see Figure 1). A social structure that is emerged from the play represents the way in which peers are connected to each other and therefore reflects on the dynamics of agents' social proxemics and group formation. Through the involvement of users in the scenario and the visualisation of individual relationships with others within the 
social structure, the VLE allows users to explicitly perceive and explore the social dynamics emerging from user-agent and agent-agent interactions.
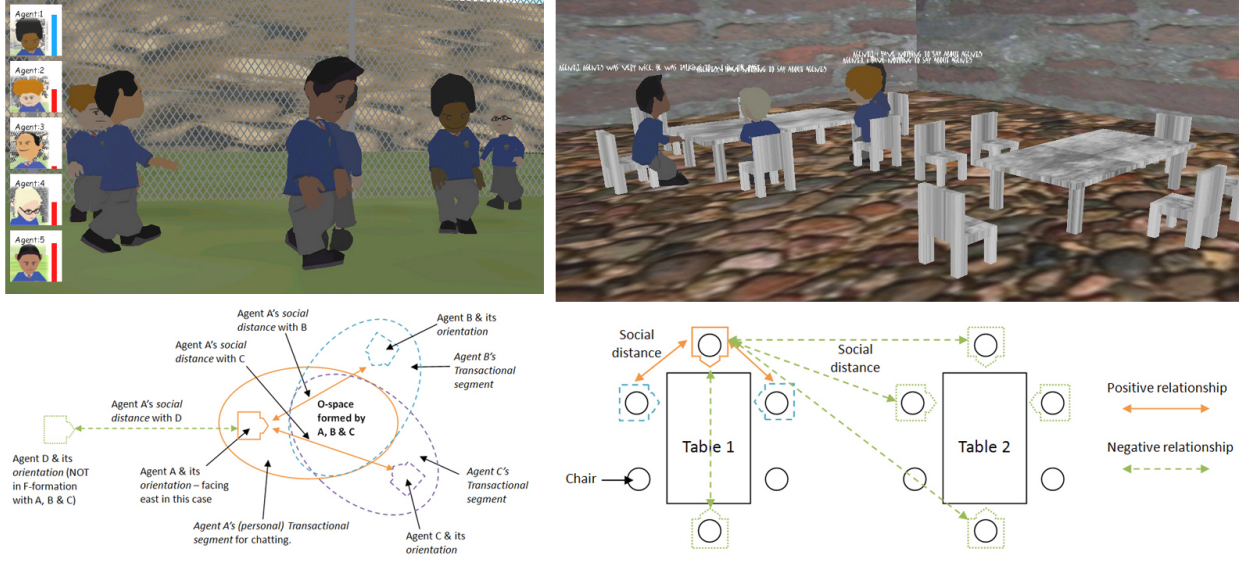

Fig. 1. Top-left: screenshot of the game interface visualising individual relationships from the user-controlled agent to others. Top-right: second part of the game scenario: Screenshot of a virtual 'canteen' with two tables. Bottom: design diagram showing that the F-formation system and social proxemics (distances maintained by one agent while staying with others) applied to agents when chatting to each other in the playground (left), and when sitting in the virtual canteen (right) - the agents' social relationships are demonstrated by their pre-defined sitting patterns.

\section{Acknowledgments}

This work was partially supported by European Community and was funded by the eCIRCUS project IST-4-027656-STP. The authors are solely responsible for the content of this publication.

\section{References}

[1] Frith, U.: Explaining the enigma. Blackwells (1989)

[2] Pedica, C., Vilhjálmsson, H.: Social perception and steering for online avatars. In: IVA '08: Proceedings of the 8th international conference on Intelligent Virtual Agents, Tokyo, Japan (2008) 104-116

[3] Kendon, A.: Spatial Organization in Social Encounters: the F-formation System. In: Studies in the behavior of social interaction. Indiana University, Bloomington and The Peter de Ridder Press, Lisse (1977) 179-208

[4] Hall, E.: The Hidden Dimension. Doubleday, New York (1966)

[5] Watson, S., Dautenhahn, K., Ho, W.C., Dawidowicz, R.: Developing relationship between autonomous agents: promoting pro-social behaviour through virtual learning environment. In Trajkovski, G.P., Collins, S.G., eds.: Handbook of Research on Agent-Based Societies: Social and Cultural Interactions. IGI Global (2009) 